Perspective

\title{
Progress in the Treatment of Migraine Attacks: From Traditional Approaches to Eptinezumab
}

\author{
Damiana Scuteri ${ }^{1,2, *(D)}$ and Giacinto Bagetta ${ }^{1, *}$ \\ 1 Pharmacotechnology Documentation and Transfer Unit, Preclinical and Translational Pharmacology, \\ Department of Pharmacy, Health and Nutritional Sciences, University of Calabria, 87036 Rende, Italy \\ 2 Regional Center for Serious Brain Injuries, S. Anna Institute, 88900 Crotone, Italy \\ * Correspondence: damiana.scuteri@unical.it (D.S.); giacinto.bagetta@unical.it (G.B.); \\ Tel.: +39-0984-493462 (D.S. \& G.B.)
}

\begin{abstract}
Migraine is the second cause of disability and of lost years of healthy life worldwide. Migraine is characterized by recurrent headache attacks and accompanying disabling symptoms lasting 4-48 h. In episodic migraine, attacks occur in less than 15 days per month and in chronic migraine, in more than 15 monthly days. Whilst successful translation of pharmacological discoveries into efficacious therapeutics has been achieved in the preventative therapy of chronic migraine, treatment of acute migraine suffers the lack of effective advancements. An effective treatment affords complete freedom from pain two hours after therapy and provides the absence of the most bothersome symptom (MBS) associated with migraine after $2 \mathrm{~h}$. However, available anti-migraine abortive treatments for acute attacks do not represent an effective and safe treatment for all the populations treated. In particular, the most used specific treatment is represented by triptans that offer 2-h sustained freedom from pain achieved in $18-50 \%$ of patients but they are contraindicated in coronary artery disease, stroke and peripheral vascular disease due to the vasoconstriction at the basis of their pharmacologic action. The most novel therapies, i.e., gepants and ditans, are without sufficient post-marketing data for secure use. Here, an attempt is proposed to analyse the rational basis and evidence in favour of investigating the efficacy and safety in acute migraine attacks of eptinezumab, i.e., monoclonal antibody $(\mathrm{mAb})$ directed towards calcitonin gene-related peptide (CGRP) unique for intravenous infusion administration.
\end{abstract}

Keywords: migraine attacks; triptans; gepants; ditans; potassium channels blockers; calcitonin gene-related peptide (CGRP); eptinezumab

D. Mitsikostas and Gill Diamond

Received: 18 August 2021

Accepted: 11 September 2021

Published: 13 September 2021

Publisher's Note: MDPI stays neutral with regard to jurisdictional claims in published maps and institutional affiliations.

\section{Introduction}

Some 716.8 million people aged $15-49$ years are affected by migraine which is the second cause of disability and of lost years of healthy life worldwide and the first within the female population [1]. The International Classification of Headache Disorders 3rd edition (ICHD-3) defines migraine as a primary headache disorder characterized by episodes which can occur with (in 1/3 of cases) or without aura, usually preceded of hours or days (premonitory phase) or associated with transient focal neurological symptoms (visual, sensory, language and brainstem disturbances) [2]. Some of these symptoms due to altered balance between sympathetic and parasympathetic systems can last over the attack to the postdromal phase [3]. Common clinical features are its unilaterality, pulsatility and moderate-to-severe intensity of throbbing pain; it is accompanied by nausea, photophobia and/or phonophobia [2]. Individual chronotypes at the basis of circadian timing of migraine attacks based on chronobiological mechanisms have been suggested [4] The fundamental relay involved in migraine pathophysiology is represented by thalamic nuclei as the core of the trigeminovascular system [5]. Nociceptive fibres from the trigeminal ganglion, especially in the ophthalmic branch of the trigeminal nerve, innervate the dura mater and the cerebral arteries. Through the release of vasoactive neuropeptides 
(e.g., calcitonin gene-related peptide, (CGRP), and pituitary adenylate cyclase-activating polypeptide, (PACAP)), ATP, glutamate and NO, the latter fibres convey painful chemical or physical stimuli from pericranial skin and muscles to second-order neurons within the trigeminal cervical complex, from which projections to the brainstem, thalamic nuclei and hypothalamus and successively from these to cortical regions originate the attack [3]. The trigeminovascular pain pathway is illustrated in Figure 1.

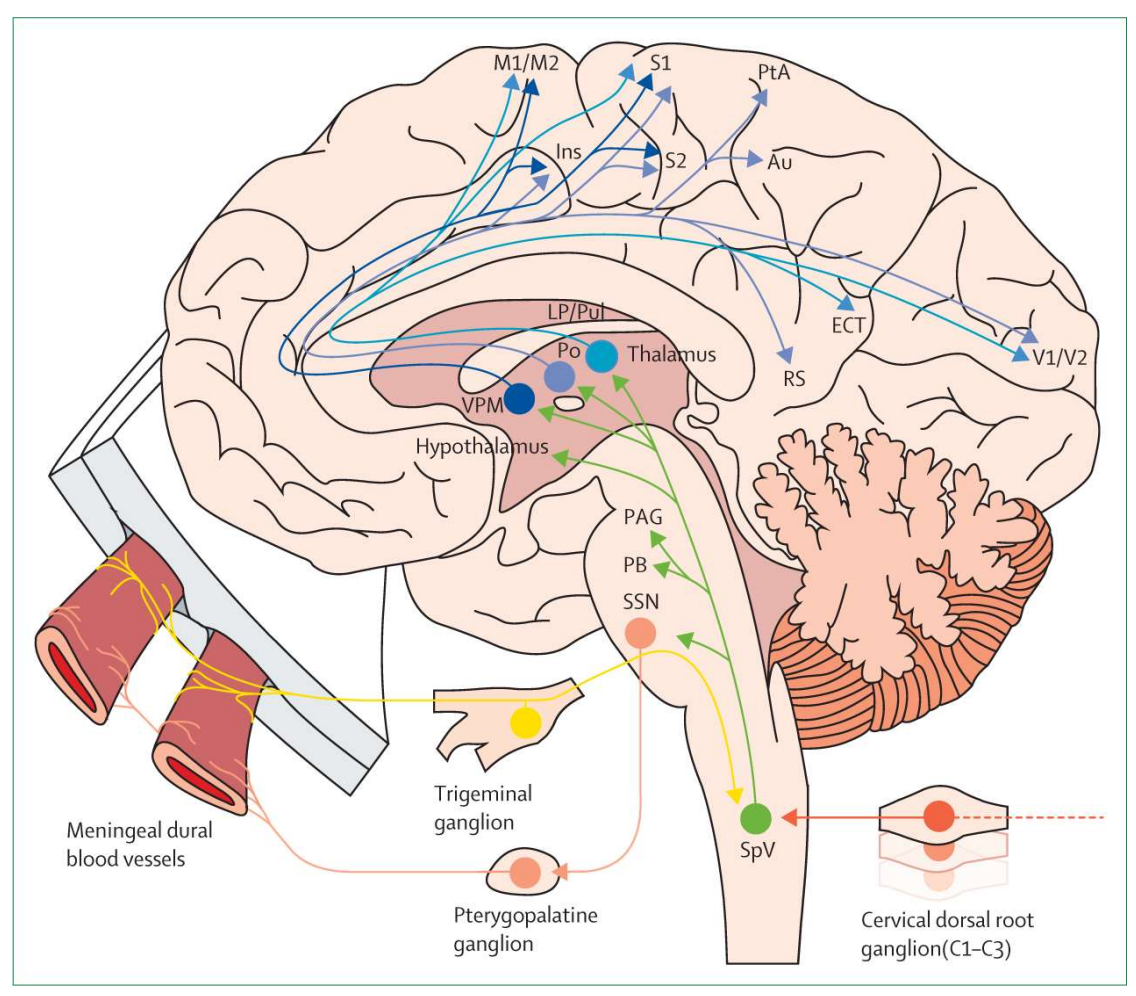

Figure 1. The complex trigeminovascular system and the activation of migraine. Sensory nociceptors innervating dural meningeal arteries undergo activation due to chemical or physical stimuli and project to second- and third-order neurons inducing migraine and sensitization. $\mathrm{Au}=\mathrm{Auditory}$ cortex. $\mathrm{ECT}=$ ectorhinal cortex. Ins = insular cortex. $\mathrm{LP}=$ lateral posterior thalamic nucleus. $\mathrm{M} 1$ = primary motor cortex. $\mathrm{M} 2$ = secondary motor cortex. $\mathrm{PAG}=$ periaqueductal gray. $\mathrm{PB}=$ parabrachial nucleus. $\mathrm{Po}=$ posterior. $\mathrm{PtA}=$ parietal association cortex. $\mathrm{Pul}=$ pulvinar. $\mathrm{RS}=$ retrosplenial cortex . $\mathrm{S} 1$ = primary somatosensory cortex. $\mathrm{S} 2=$ secondary somatosensory cortex. $\mathrm{SpV}=$ spinal trigeminal nucleus. SSN = superior salivatory nucleus. $\mathrm{V} 1$ = primary visual cortex. $\mathrm{V} 2$ = secondary visual cortex. $\mathrm{VPM}=$ ventral posteromedial (adapted with permission from [6]).

\section{Therapies for Migraine Attacks and Unmet Medical Needs}

According to the International Headache Society (IHS) recommendations for the clinical trials, testing pharmacological agents for the acute treatment of migraine, the testing agents needs to provide $2 \mathrm{~h}$ after therapy complete freedom from pain and absence of the most bothersome symptom (MBS) associated to migraine [7]. Therefore, the ideal treatment of migraine attacks should provide the highest speed of action onset against pain and the MBS, which should be long-lasting to prevent relapse within 24 or $48 \mathrm{~h}$ from the initial treatment (sustained pain freedom), together with a good safety profile allowing for adherence. Currently, the horizon of evidence-based analgesics for the acute treatment of migraine has expanded but the complete and safe relief from acute attacks in a high percentage of patients is still an unmet medical need. Novel acute treatments are added to the established therapeutic armamentarium that can be distinguished in non-specific, symptomatic treatments and specific anti-migraine drugs for the abortion of acute attacks. 


\subsection{Not Specific Symptomatic Treatments}

Acetaminophen and non-steroidal anti-inflammatory drugs (NSAIDs), in particular aspirin, diclofenac, and ibuprofen, represent the first-line treatment for mild-to-moderate acute episodes. All the NSAIDs have met the primary endpoint of the disappearance of pain after $2 \mathrm{~h}$, but in a small percentage of patients compared to placebo [8,9]. A Cochrane systematic review and its update have demonstrated that ibuprofen confers relief in half of the population, but the complete disappearance of headache pain and symptoms only in a minority of migraineurs $[10,11]$. Additionally, diclofenac provides pain-free time only in a minority of patients [12]. Aspirin has resulted effective for acute migraine sufferers, but for the reduction of the symptoms nausea and vomiting the addition of metoclopramide $10 \mathrm{mg}$ is useful $[13,14]$. Naproxen has proven effective in only 2 out of 10 migraine sufferers, not being a stand-alone clinically useful treatment [15]. Another important issue of NSAIDs is represented by the adverse reactions, including gastrointestinal and cardiovascular serious side effects also induced by cyclooxygenase 2 (COX-2) inhibitors. In fact, the PRECISION-ABPM (Prospective Randomized Evaluation of Celecoxib Integrated Safety vs. Ibuprofen or Naproxen Ambulatory Blood Pressure Measurement) Trial has demonstrated hypertension in $23.2 \%$ patients for ibuprofen, $19.0 \%$ for naproxen and $10.3 \%$ for celecoxib with physiological baseline blood pressure values [16]. Among the most ancient therapies for migraine, the ergot derivatives assume a historical significance, leading to the development of dihydroergotamine now available as injectable (intravenous/intramuscular/subcutaneous) and intranasal formulations [17]. The use of the latter drug is not feasible without an anti-emetic and in patients with cardiovascular disease and hypertension. In fact, they are characterized by an increased rate of catheterassociated venous thromboses [18] and a case of likely vasospastic angina in the absence of coronary artery disease has been described [19]. In course of severe migraine attacks, also opiates can be used, paying attention to the mortality rate for abuse of these drugs often used in combination with gabapentinoids [20].

\subsection{Anti-Migraine Abortive Treatments for Acute Attacks}

Triptans are selective $5-\mathrm{HT}_{1 \mathrm{~B} / 1 \mathrm{D} /(1 \mathrm{~F})}$ agonists used as a gold standard treatment for moderate-to-severe acute migraine attacks. Unfortunately, some $40 \%$ of migraine sufferers do not respond to the latter treatment and percentages of complete pain relief after $2 \mathrm{~h}$ are variable, as it is the tolerability profile within the class [21,22]. In fact, the first triptan to be used since the early 1990 's, sumatriptan, is characterized by poor oral bioavailability, $\sim 14 \%$ ( $100 \%$ after subcutaneous administration) and lipophilicity [23], thus leading to zolmitriptan, eletriptan, frovatriptan, rizatriptan and almotriptan, the second generation with improved pharmacokinetics (PK) [24]. Among these, almotriptan is one of the newest triptan with a favourable PK profile, $70 \%$ oral bioavailability, not affected by gender or food [25], and not bearing important drug-to-drug metabolic interactions apart from with CYP3A4 inhibitors [24]. These characteristics may in part explain its frequency of use in real-world settings [26]. Intranasal zolmitriptan presents fast cerebral penetration and PK advantages over the other routes of administration [27]. In fact, a nasal spray is available for sumatriptan and for zolmitriptan in Italy [28]. A network meta-analysis has compared the effectiveness of the various triptans and of not specific symptomatic treatments [29]. Based on this study, triptans afforded 2-h sustained freedom from pain in $18-50 \%$ of patients [29]. Data of pain relief and freedom at $2 \mathrm{~h}$ for each triptan and for the other symptomatic treatments [29] are reported in Table 1. 
Table 1. Percentage of Patients, Odds ratio (OR) and 95\% Credible Interval (CrI) with headache relief or freedom from pain at $2 \mathrm{~h}$ with a standard dose of triptans and not specific symptomatic treatments administered for oral route.

\begin{tabular}{lcc}
\hline \multicolumn{1}{c}{ Drug (Standard Dose) } & 2-h Headache Relief & 2-h Freedom from Pain \\
\hline Almotriptan (12.5 mg) & $48.30 \%, 2.56(2.0-3.3)$ & $24.50 \%, 2.73(2.1-3.6)$ \\
Eletriptan (40 mg) & $60.40 \%, 4.19(3.5-5.0)$ & $39.20 \%, 5.43(4.3-6.9)$ \\
Frovatriptan (2.5 mg) & $42.40 \%, 2.02(1.5-2.7)$ & $34.70 \%, 4.48(2.8-7.3)$ \\
Naratriptan $(2.5 \mathrm{mg})$ & $44.50 \%, 2.20(1.5-3.2)$ & $17.50 \%, 1.79(1.1-2.8)$ \\
Rizatriptan (10 mg) & $57.10 \%, 3.66(3.0-4.5)$ & $36.60 \%, 4.86(3.9-6.2)$ \\
Sumatriptan (50 mg) & $49.70 \%, 2.71(2.4-3.1)$ & $27.70 \%, 3.22(2.7-3.8)$ \\
Zolmitriptan (2.5 mg) & $50.00 \%, 2.75(2.3-3.3)$ & $27.10 \%, 3.14(2.5-4.0)$ \\
Acetaminophen $(1000 \mathrm{mg})$ & $51.70 \%, 2.94(1.2-7.2)$ & $22.20 \%, 2.41(0.9-6.6)$ \\
Aspirin (1000 $\mathrm{mg})$ & $46.10 \%, 2.35(1.3-4.2)$ & $24.00 \%, 2.66(1.3-5.3)$ \\
Ergot derivatives $(2 \mathrm{mg})$ & $38.40 \%, 1.71(1.2-2.5)$ & $15.50 \%, 1.55(0.8-3.2)$ \\
\hline
\end{tabular}

Apart from the $40 \%$ of triptan users that reported unmet needs [30], it is very important to underline that triptans exert their therapeutic action through the vasoconstriction of cranial vessels (via agonism at $5-\mathrm{HT}_{1 \mathrm{~B}}$ receptor and an indirect effect on CGRP), consequently inhibiting transmission throughout the trigeminocervical system. This characteristic makes them contraindicated with coronary artery disease, stroke, and peripheral vascular diseases [31]. Therefore, the research has moved towards drugs that do not induce vasoconstriction, i.e., gepants that are CGRP receptor antagonists and ditans that are $5-\mathrm{HT}_{1 \mathrm{~F}}$ receptor agonists. Since these drugs have been recently approved, longer clinical postmarketing experience is needed for the accurate determination of adverse events, mainly for people suffering from cardiovascular diseases and during pregnancy [32]. Ubrogepant has been investigated in the NCT03461757 double-blind, randomised, placebo-controlled, multicentre phase III trial [33]; in the NCT03237845 multicentre, double-blind, phase III clinical trial [34]; in the "Efficacy, Safety, and Tolerability Study of Oral Ubrogepant in the Acute Treatment of Migraine" (ACHIEVE I) (NCT02828020) phase III, multicentre, randomized, double-blind, placebo-controlled single attack study [35] and in the phase III, multicentre, randomized, double-blind, placebo-controlled, single-attack, (ACHIEVE II) (NCT02867709) [36], it was discovered that pain freedom occured at $2 \mathrm{~h}$ in 19.2-21.8\% of patients, based on the dose, and freedom from the migraine-associated MBS at $2 \mathrm{~h}$ in $34.1-38.9 \%$ of patients. Data for rimegepant efficacy obtained from the double-blind, randomised, placebo-controlled, multicentre phase III trial NCT03461757 [33] and from the multicentre, double-blind, phase III clinical trial NCT03237845 [34] demonstrated that some $19.6-21 \%$ patients achieved pain freedom at $2 \mathrm{~h}$ and some $35.0-37.6 \%$ obtained freedom from the migraine-associated MBS. Lasmiditan is the first ditan to be used and it has demonstrated efficacy in terms of providing pain freedom at $2 \mathrm{~h}$ in $10.0-32.2 \%$ of patients with $11.1-40.9 \%$ patients achieving freedom from migraine-associated MBS at $2 \mathrm{~h}[37,38]$. In fact, it has provided significant efficacy, meeting key primary and secondary efficacy endpoints at $2 \mathrm{~h}$ in two randomized, double-blind, placebo-controlled, phase III clinical trials, that are "A Study of Two Doses of LAsMiditan (100 mg and $200 \mathrm{mg}$ ) Compared to Placebo in the AcUte Treatment of MigRAIne" (SAMURAI) (NCT02439320) and "A Study of Three Doses of Lasmiditan (50 mg, $100 \mathrm{mg}$ and $200 \mathrm{mg}$ ) Compared to Placebo in the Acute TReaTment of MigrAiNe" (SPARTAN) (NCT02605174) in adults without aging limitations $[37,38]$. The following phase III, randomized, open-label, multi-attack study, "An Open-label, LonG-term, Safety Study of LAsmiDItan (100 mg and $200 \mathrm{mg}$ ) in the Acute Treatment Of MigRaine" (GLADIATOR) (NCT02565186) [39], has investigated the incidence of treatment-emergent adverse events (TEAEs) in 132 elderly subjects enrolled to the treatment (54 to placebo) from SAMURAI and SPARTAN trials and also had 85 elderly subjects from GLADIATOR itself since this population is often excluded from migraine studies. Comparability of TEAEs incidence in elderly and non-elderly has been observed in this limited sample, with at least one TEAE occurring in $36 \%$ and $35 \%$ in 
pooled SAMURAI + SPARTAN and in 49\% and 38\% in GLADIATOR of non-elderly and elderly, respectively [39]. Therefore, future studies are needed to investigate the durability of the effectiveness and the long-term safety of gepants and ditans in the different patient populations, particularly those suffering from cardiovascular diseases and in pregnancy, in comparison with other drugs.

\section{Novel Perspectives and Future Directions: Focus on Eptinezumab}

The therapeutic options described above demonstrate, with a large degree of strength, the evidence of the efficacy (limited only for opioids [40]) and the great translational success of the research effort of the last 30 years; however, a safe abortive treatment for acute attacks effective in most patients is still lacking. Recently, potassium channels have gained interest in the field of migraine. Among migraine attack triggering molecules, e.g., CGRP, drugs that open large-conductance calcium-activated potassium $\left(\mathrm{BK}_{\mathrm{Ca}}\right)$ and ATP-sensitive potassium $\left(\mathrm{K}_{\mathrm{ATP}}\right)$ channels $[41,42]$ on vascular smooth muscles cells of intracranial arteries have been recently identified as potential inducers of potassium efflux with following vasodilation and signalling of trigeminal nociceptors [43]. While further studies explore the role of the blockers of these channels, the story of monoclonal antibodies (mAbs) directed towards CGRP (eptinezumab, fremanezumab, and galcanezumab) and its canonical receptor (erenumab) has not come to an end and therapeutic innovation can be anticipated. In fact, mAbs have revolutionized the therapy of several neurologic diseases, acting through direct mechanisms in the case of migraine [44]. The presence of CGRP immunoreactive fibres around cerebral vessels originating from the trigeminal ganglion has been demonstrated (Figure 2) shedding light on the neurogenic activities exerted by the trigemino-cerebrovascular system [45].
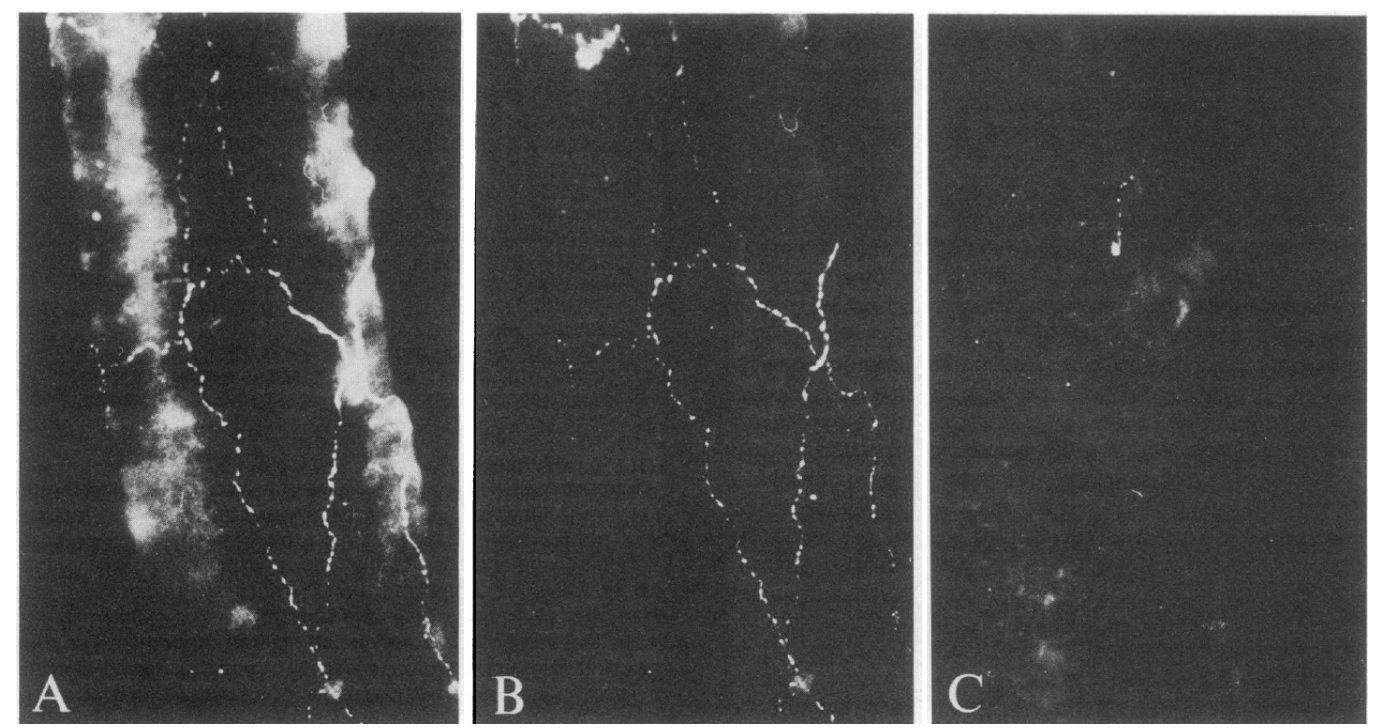

Figure 2. The immunoreactivity of calcitonin gene-related peptide (CGRP) around cerebral blood vessels. (A) Network of CGRP immunofluorescence in fibres innervating the cerebral arterioles. (B) Colocalization with substance P (SP). (C) Almost complete absence of CGRP immunofluorescence in cerebral arterioles after ipsilateral trigeminal nerve surgical lesion 14 days before sacrifice (adapted with permission from [45]).

However, the site of action of anti-CGRP mAbs, peripheral or intracerebral, is still a debated issue though there is pharmacological evidence in support of a central action [46]; the latter strengthens the need for deepened investigation to understand the therapeutic potential of the latter biologics. Erenumab efficacy has been studied in the randomized, doubleblind, placebo-controlled, phase III "Study to Evaluate the Efficacy and Safety of Erenumab (AMG 334) Compared to Placebo in Migraine Prevention (ARISE)" (NCT02483585) [47], showing significant differences with the placebo in monthly migraine days (MMDs) and 
a $\geq 50 \%$ reduction in $39.7 \%$, with the typical side effects of these treatments most frequently consisting in upper respiratory tract infections and injection site pain. The phase III, randomized, double-blind, placebo-controlled "Study to Evaluate the Efficacy and Safety of Erenumab (AMG 334) in Migraine Prevention" (STRIVE) (NCT02456740) [48] has highlighted a 3.2-3.7 dose-related MMDs reduction. The efficacy of erenumab in episodic migraine non-responding to previous treatments has been assessed in 12 week-phase IIIb "Study Evaluating the Effectiveness of AMG 334 Injection in Preventing Migraines in Adults Having Failed Other Therapies" (LIBERTY) (NCT03096834) [49] reporting its efficacy in these difficult-to-treat patients. The effectiveness in episodic (HALO EM, NCT02629861) and chronic (HALO CM) migraine has been observed also for fremanezumab, even in the reduction of acute medications [50-52]. Additionally, efficacy in patients whose treatments had previously failed has been documented for fremanezumab in the phase IIIb multicentre, randomized, double-blind, parallel-group, placebo-controlled (with an open-label period) "Efficacy and Safety Study of Fremanezumab in Adults With Migraine" (FOCUS) (NCT03308968) study [53]. Moreover, the 52-week, multicentre, randomized, double-blind, parallel-group study (NCT02638103) has confirmed the long-term safety and tolerability of fremanezumab [54]. Additionally, galcanezumab has provided evidence for efficacy in the prevention of episodic migraine in the "Evaluation of LY2951742 in the Prevention of Episodic Migraine 1 and 2" (EVOLVE-1 and EVOLVE-2) double-blind, randomized, placebo-controlled trials (NCT02614183, NCT02614196) $[55,56]$ and of chronic migraine in the phase III "Evaluation of Galcanezumab in the Prevention of Chronic Migraine" (REGAIN) study (NCT02614261) [57], showing long-term safety in the phase III, long-term, open-label NCT02614287 safety study [58], and has shown some reduced efficacy posttreatment [59]. The effectiveness of fremanezumab in treatment-resistant migraine has been assessed in patients aged 18-75 years in the "Study of Galcanezumab (LY2951742) in Adults With Treatment-Resistant Migraine (CONQUER)" (NCT03559257) [60]. The phase III, randomized, double-blind, placebo-controlled Prevention of Migraine via Intravenous ALD403 Safety and Efficacy 1 (PROMISE-1) (NCT02559895) clinical trial [61] has demonstrated the significant effectiveness of the intravenous administration of eptinezumab with a higher number of patients with a $\geq 50 \%$ or $\geq 75 \%$ reduction of episodic migraine compared to the placebo during one year of treatment, in which it was well tolerated. The phase III, randomized, double-blind, placebo-controlled PROMISE-2 (NCT02974153) study has demonstrated significant improvement and preventative efficacy in patients suffering from chronic migraine in 24 weeks [62]. The long-term (2 year-long) safety of eptinezumab in patients with chronic migraine has been evaluated in the study, "An Open Label Trial of ALD403 (Eptinezumab) in Chronic Migraine" (PREVAIL) (NCT02985398) [63], which has confirmed the benefit in chronic migraine associated with a favourable safety profile 24 week-immunogenicity up to non-detectable levels. The PK characteristics of anti-CGRP mAbs may account for some differences in their therapeutic application. In fact, eptinezumab is the only anti-CGRP $\mathrm{mAb}$ administered for intravenous infusion adding a faster action [64] due to the rapid reaching of maximum plasma concentration (Cmax; within around $30 \mathrm{~min}$, i.e., the end of the infusion) [65,66], to the long half-life (characteristic of the CGRP mAbs class of biologics), allowing the dilated frequency of administration [67]. In particular, eptinezumab intravenous administration occurs every 3 months and it has demonstrated a promising preventive effect in episodic migraine [68]. The phase 3, multicentre, parallel-group, double-blind, randomized, placebo-controlled trial NCT04152083 [69] has assessed the efficacy of the infusion of eptinezumab during an active migraine attack in patients eligible for preventive migraine treatment based on ICHD-3 criteria: the co-primary endpoints were time to headache pain freedom and time to absence of MBS, both expressed as median time. The results of this study are noteworthy since eptinezumab provided headache pain freedom $4 \mathrm{~h}$ after the start of infusion and the absence of MBS $2 \mathrm{~h}$ after the start of infusion [69]. Furthermore, eptinezumab has been found to afford faster time to headache pain relief, sustained headache pain freedom and delayed time to next attack with a favourable safety profile [69]. Status migrainosus 
is a debilitating attack lasting longer than $72 \mathrm{~h}$, usually treated with parenteral drugs (e.g., ketorolac or dihydroergotamine); eptinezumab could be very useful for its rapid action and its preventative efficacy. In fact, anti-CGRP mAbs show a better benefit/risk ratio in comparison with established therapies for episodic and chronic migraine [70]. Therefore, although not approved for acute treatment, eptinezumab is endowed with the potential to abort migraine attacks: these data, in conjunction with the PK characteristics, do support the need for a future clinical trial designed to investigate the efficacy and safety of the latter $\mathrm{mAb}$ in the treatment of acute attacks occurring 2-8 times per month with at least $48 \mathrm{~h}$ of pain freedom between attacks and fewer than 15 headache days per month, according to current criteria for acute attacks clinical trials [7].

\section{Conclusions}

The search for an effective and safe abortive treatment for migraine attacks affording efficacy to most sufferers still represents a challenge. In fact, triptans provide 2-h of sustained freedom from pain, this was observed in $18-50 \%$ patients [29] and the most novel options, i.e., gepants and ditans, need long-term post-marketing reporting of data for adverse events in people affected by cardiovascular diseases and in pregnant women [32] Pharmacologic [64] and clinical data (see NCT04152083 clinical trial) [69] form the rational basis for the study of the latter anti-CGRP $\mathrm{mAb}$, being administered for intravenous infusion and reaching $\mathrm{Cmax}$ at the end of the infusion, in acute attacks. Therefore, a clinical trial with the adequate design to investigate the efficacy and safety of eptinezumab in the treatment of acute attacks occurring 2-8 times per month with at least $48 \mathrm{~h}$ of pain freedom between attacks and fewer than 15 headache days per month [7] is needed. Moreover, due to the epidemiology of migraine, generally the eldest population are not included in clinical trials for current abortive treatments, e.g., triptans [26]: this issue is worsened in patients affected by dementia who do not receive adequate treatment for chronic pain [71-73]. Hence, this clinical trial should include this fragile population. Another pivotal problem is represented by patients suffering from mild traumatic brain injury who can develop post-traumatic headache in which the pathogenesis involves CGRP as well [74]. The investigation of eptinezumab could represent a completely new pattern of treatment for acute attacks, including effective prevention of following episodes.

Author Contributions: Conceptualization, D.S. and G.B.; methodology, D.S. and G.B.; investigation, D.S. and G.B.; writing-review and editing, D.S. and G.B. All authors have read and agreed to the published version of the manuscript.

Funding: This research received no external funding.

Institutional Review Board Statement: Not applicable.

Informed Consent Statement: Not applicable.

Data Availability Statement: No new data were created or analysed in this study. Data sharing is not applicable to this article.

Acknowledgments: D.S. is a post-doc funded by S. Anna Institute, Crotone, Italy.

Conflicts of Interest: The authors declare no conflict of interest.

\section{References}

1. Steiner, T.J.; Stovner, L.J.; Jensen, R.; Uluduz, D.; Katsarava, Z.; On behalf of Lifting The Burden: The Global Campaign Headache. Migraine remains second among the world's causes of disability, and first among young women: Findings from GBD2019. J. Headache Pain 2020, 21, 137. [CrossRef]

2. Headache Classification Committee of the International Headache Society (IHS) The International Classification of Headache Disorders, 3rd edition. Cephalalgia 2018, 38, 1-211. [CrossRef]

3. Dodick, D.W. A Phase-by-Phase Review of Migraine Pathophysiology. Headache 2018, 58 (Suppl. 1), 4-16. [CrossRef]

4. Van Oosterhout, W.; Van Someren, E.; Schoonman, G.G.; Louter, M.A.; Lammers, G.J.; Ferrari, M.D.; Terwindt, G.M. Chronotypes and circadian timing in migraine. Cephalalgia 2018, 38, 617-625. [CrossRef] 
5. Younis, S.; Hougaard, A.; Noseda, R.; Ashina, M. Current understanding of thalamic structure and function in migraine. Cephalalgia 2019, 39, 1675-1682. [CrossRef]

6. Ashina, M.; Hansen, J.M.; Do, T.P.; Melo-Carrillo, A.; Burstein, R.; Moskowitz, M.A. Migraine and the trigeminovascular system-40 years and counting. Lancet Neurol. 2019, 18, 795-804. [CrossRef]

7. Diener, H.C.; Tassorelli, C.; Dodick, D.W.; Silberstein, S.D.; Lipton, R.B.; Ashina, M.; Becker, W.J.; Ferrari, M.D.; Goadsby, P.J.; Pozo-Rosich, P.; et al. Guidelines of the International Headache Society for controlled trials of acute treatment of migraine attacks in adults: Fourth edition. Cephalalgia 2019, 39, 687-710. [CrossRef] [PubMed]

8. MacGregor, E.A.; Dowson, A.; Davies, P.T. Mouth-dispersible aspirin in the treatment of migraine: A placebo-controlled study. Headache 2002, 42, 249-255. [CrossRef] [PubMed]

9. Codispoti, J.R.; Prior, M.J.; Fu, M.; Harte, C.M.; Nelson, E.B. Efficacy of nonprescription doses of ibuprofen for treating migraine headache. a randomized controlled trial. Headache 2001, 41, 665-679. [CrossRef] [PubMed]

10. Rabbie, R.; Derry, S.; Moore, R.A.; McQuay, H.J. Ibuprofen with or without an antiemetic for acute migraine headaches in adults. Cochrane Database Syst. Rev. 2010, Cd008039. [CrossRef]

11. Rabbie, R.; Derry, S.; Moore, R.A. Ibuprofen with or without an antiemetic for acute migraine headaches in adults. Cochrane Database Syst. Rev. 2013, 2013, Cd008039. [CrossRef] [PubMed]

12. Derry, S.; Rabbie, R.; Moore, R.A. Diclofenac with or without an antiemetic for acute migraine headaches in adults. Cochrane Database Syst. Rev. 2013, 2013, Cd008783. [CrossRef]

13. Kirthi, V.; Derry, S.; Moore, R.A.; McQuay, H.J. Aspirin with or without an antiemetic for acute migraine headaches in adults. Cochrane Database Syst. Rev. 2010, Cd008041. [CrossRef]

14. Kirthi, V.; Derry, S.; Moore, R.A. Aspirin with or without an antiemetic for acute migraine headaches in adults. Cochrane Database Syst. Rev. 2013, 2013, Cd008041. [CrossRef] [PubMed]

15. Law, S.; Derry, S.; Moore, R.A. Naproxen with or without an antiemetic for acute migraine headaches in adults. Cochrane Database Syst. Rev. 2013, 2013, Cd009455. [CrossRef]

16. Ruschitzka, F.; Borer, J.S.; Krum, H.; Flammer, A.J.; Yeomans, N.D.; Libby, P.; Lüscher, T.F.; Solomon, D.H.; Husni, M.E.; Graham, D.Y.; et al. Differential blood pressure effects of ibuprofen, naproxen, and celecoxib in patients with arthritis: The PRECISIONABPM (Prospective Randomized Evaluation of Celecoxib Integrated Safety Versus Ibuprofen or Naproxen Ambulatory Blood Pressure Measurement) Trial. Eur. Heart J. 2017, 38, 3282-3292. [CrossRef]

17. Silberstein, S.D.; Shrewsbury, S.B.; Hoekman, J. Dihydroergotamine (DHE)—Then and Now: A Narrative Review. Headache 2020, 60, 40-57. [CrossRef] [PubMed]

18. Tso, A.R.; Patniyot, I.R.; Gelfand, A.A.; Goadsby, P.J. Increased rate of venous thrombosis may be associated with inpatient dihydroergotamine treatment. Neurology 2017, 89, 279-283. [CrossRef]

19. Schenkat, D.H.; Schulz, L.T.; Johnson, B.D. Dihydroergotamine-induced vasospastic angina in a patient taking a calcium channel blocker. Ann. Pharmacother. 2011, 45, e41. [CrossRef]

20. Eastwood, J.A.; Davison, E. Pregabalin concentrations in post-mortem blood-A two year study. Forensic Sci. Int. 2016, 266, 197-201. [CrossRef]

21. Ferrari, M.D.; Roon, K.I.; Lipton, R.B.; Goadsby, P.J. Oral triptans (serotonin 5-HT(1B/1D) agonists) in acute migraine treatment: A meta-analysis of 53 trials. Lancet 2001, 358, 1668-1675. [CrossRef]

22. Edvinsson, L. CGRP receptor antagonists and antibodies against CGRP and its receptor in migraine treatment. Br. J. Clin. Pharmacol. 2015, 80, 193-199. [CrossRef]

23. Fowler, P.A.; Lacey, L.F.; Thomas, M.; Keene, O.N.; Tanner, R.J.N.; Baber, N.S. The Clinical Pharmacology, Pharmacokinetics and Metabolism of Sumatriptan. Eur. Neurol. 1991, 31, 291-294. [CrossRef]

24. Negro, A.; Koverech, A.; Martelletti, P. Serotonin receptor agonists in the acute treatment of migraine: A review on their therapeutic potential. J. Pain Res. 2018, 11, 515-526. [CrossRef]

25. Dahlöf, C.G.; Dodick, D.; Dowson, A.J.; Pascual, J. How does almotriptan compare with other triptans? A review of data from placebo-controlled clinical trials. Headache 2002, 42, 99-113. [CrossRef] [PubMed]

26. Scuteri, D.; Adornetto, A.; Rombolà, L.; Naturale, M.D.; De Francesco, A.E.; Esposito, S.; Zito, M.; Morrone, L.A.; Bagetta, G.; Tonin, P.; et al. Pattern of triptans use: A retrospective prescription study in Calabria, Italy. Neural Regen. Res. 2020, 15, 1340-1343. [CrossRef] [PubMed]

27. Goadsby, P.J.; Yates, R. Zolmitriptan intranasal: A review of the pharmacokinetics and clinical efficacy. Headache 2006, 46, 138-149. [CrossRef] [PubMed]

28. Marmura, M.J.; Silberstein, S.D.; Schwedt, T.J. The acute treatment of migraine in adults: The american headache society evidence assessment of migraine pharmacotherapies. Headache 2015, 55, 3-20. [CrossRef]

29. Cameron, C.; Kelly, S.; Hsieh, S.C.; Murphy, M.; Chen, L.; Kotb, A.; Peterson, J.; Coyle, D.; Skidmore, B.; Gomes, T.; et al. Triptans in the Acute Treatment of Migraine: A Systematic Review and Network Meta-Analysis. Headache 2015, 55 (Suppl. 4), $221-235$. [CrossRef] [PubMed]

30. Piccinni, C.; Cevoli, S.; Ronconi, G.; Dondi, L.; Calabria, S.; Pedrini, A.; Esposito, I.; Favoni, V.; Pierangeli, G.; Cortelli, P.; et al. A real-world study on unmet medical needs in triptan-treated migraine: Prevalence, preventive therapies and triptan use modification from a large Italian population along two years. J. Headache Pain 2019, 20, 74. [CrossRef] 
31. Mathew, P.G.; Klein, B.C. Getting to the Heart of the Matter: Migraine, Triptans, DHE, Ditans, CGRP Antibodies, First/SecondGeneration Gepants, and Cardiovascular Risk. Headache 2019, 59, 1421-1426. [CrossRef]

32. Negro, A.; Martelletti, P. Gepants for the treatment of migraine. Expert Opin. Investig. Drugs 2019, 28, 555-567. [CrossRef] [PubMed]

33. Croop, R.; Goadsby, P.J.; Stock, D.A.; Conway, C.M.; Forshaw, M.; Stock, E.G.; Coric, V.; Lipton, R.B. Efficacy, safety, and tolerability of rimegepant orally disintegrating tablet for the acute treatment of migraine: A randomised, phase 3, double-blind, placebo-controlled trial. Lancet 2019, 394, 737-745. [CrossRef]

34. Lipton, R.B.; Croop, R.; Stock, E.G.; Stock, D.A.; Morris, B.A.; Frost, M.; Dubowchik, G.M.; Conway, C.M.; Coric, V.; Goadsby, P.J. Rimegepant, an Oral Calcitonin Gene-Related Peptide Receptor Antagonist, for Migraine. N. Engl. J. Med. 2019, 381, 142-149. [CrossRef] [PubMed]

35. Dodick, D.W.; Lipton, R.B.; Ailani, J.; Lu, K.; Finnegan, M.; Trugman, J.M.; Szegedi, A. Ubrogepant for the Treatment of Migraine. N. Engl. J. Med. 2019, 381, 2230-2241. [CrossRef]

36. Lipton, R.B.; Dodick, D.W.; Ailani, J.; Lu, K.; Finnegan, M.; Szegedi, A.; Trugman, J.M. Effect of Ubrogepant vs Placebo on Pain and the Most Bothersome Associated Symptom in the Acute Treatment of Migraine: The ACHIEVE II Randomized Clinical Trial. JAMA 2019, 322, 1887-1898. [CrossRef] [PubMed]

37. Ashina, M.; Vasudeva, R.; Jin, L.; Lombard, L.; Gray, E.; Doty, E.G.; Yunes-Medina, L.; Kinchen, K.S.; Tassorelli, C. Onset of Efficacy Following Oral Treatment With Lasmiditan for the Acute Treatment of Migraine: Integrated Results From 2 Randomized Double-Blind Placebo-Controlled Phase 3 Clinical Studies. Headache 2019, 59, 1788-1801. [CrossRef] [PubMed]

38. Kuca, B.; Silberstein, S.D.; Wietecha, L.; Berg, P.H.; Dozier, G.; Lipton, R.B. Lasmiditan is an effective acute treatment for migraine: A phase 3 randomized study. Neurology 2018, 91, e2222-e2232. [CrossRef]

39. Martin, V.T.; Ahmed, Z.; Hochstetler, H.M.; Baygani, S.K.; Dong, Y.; Hauck, P.M.; Khanna, R. Tolerability and Safety of Lasmiditan Treatment in Elderly Patients With Migraine: Post Hoc Analyses From Randomized Studies. Clin. Ther. 2021. [CrossRef]

40. VanderPluym, J.H.; Halker Singh, R.B.; Urtecho, M.; Morrow, A.S.; Nayfeh, T.; Torres Roldan, V.D.; Farah, M.H.; Hasan, B.; Saadi, S.; Shah, S.; et al. Acute Treatments for Episodic Migraine in Adults: A Systematic Review and Meta-analysis. JAMA 2021, 325, 2357-2369. [CrossRef]

41. Al-Karagholi, M.A.-M.; Ghanizada, H.; Waldorff Nielsen, C.A.; Skandarioon, C.; Snellman, J.; Lopez-Lopez, C.; Hansen, J.M.; Ashina, M. Opening of BKCa channels causes migraine attacks: A new downstream target for the treatment of migraine. Pain 2021. [CrossRef]

42. Al-Karagholi, M.A.-M.; Ghanizada, H.; Nielsen, C.A.W.; Hougaard, A.; Ashina, M. Opening of ATP sensitive potassium channels causes migraine attacks with aura. Brain J. Neurol. 2021. [CrossRef]

43. Rapoport, A.M.; Lipton, R.B. Potassium channel openers-Novel triggers of aura and migraine. Nat. Rev. Neurol. 2021, 17, 397-398. [CrossRef] [PubMed]

44. Gklinos, P.; Papadopoulou, M.; Stanulovic, V.; Mitsikostas, D.D.; Papadopoulos, D. Monoclonal Antibodies as Neurological Therapeutics. Pharmaceuticals 2021, 14, 92. [CrossRef] [PubMed]

45. McCulloch, J.; Uddman, R.; Kingman, T.A.; Edvinsson, L. Calcitonin gene-related peptide: Functional role in cerebrovascular regulation. Proc. Natl. Acad. Sci. USA 1986, 83, 5731-5735. [CrossRef] [PubMed]

46. Chiarugi, A. A Popperian View on Anti-CGRP Biologics in Migraine. Headache J. Head Face Pain 2019, 59, 1855-1860. [CrossRef]

47. Dodick, D.W.; Ashina, M.; Brandes, J.L.; Kudrow, D.; Lanteri-Minet, M.; Osipova, V.; Palmer, K.; Picard, H.; Mikol, D.D.; Lenz, R.A. ARISE: A Phase 3 randomized trial of erenumab for episodic migraine. Cephalalgia 2018, 38, 1026-1037. [CrossRef]

48. Goadsby, P.J.; Reuter, U.; Hallström, Y.; Broessner, G.; Bonner, J.H.; Zhang, F.; Sapra, S.; Picard, H.; Mikol, D.D.; Lenz, R.A. A Controlled Trial of Erenumab for Episodic Migraine. N. Engl. J. Med. 2017, 377, 2123-2132. [CrossRef]

49. Reuter, U.; Goadsby, P.J.; Lanteri-Minet, M.; Wen, S.; Hours-Zesiger, P.; Ferrari, M.D.; Klatt, J. Efficacy and tolerability of erenumab in patients with episodic migraine in whom two-to-four previous preventive treatments were unsuccessful: A randomised, double-blind, placebo-controlled, phase 3b study. Lancet 2018, 392, 2280-2287. [CrossRef]

50. Dodick, D.W.; Silberstein, S.D.; Bigal, M.E.; Yeung, P.P.; Goadsby, P.J.; Blankenbiller, T.; Grozinski-Wolff, M.; Yang, R.; Ma, Y.; Aycardi, E. Effect of Fremanezumab Compared With Placebo for Prevention of Episodic Migraine: A Randomized Clinical Trial. JAMA 2018, 319, 1999-2008. [CrossRef]

51. Silberstein, S.D.; Dodick, D.W.; Bigal, M.E.; Yeung, P.P.; Goadsby, P.J.; Blankenbiller, T.; Grozinski-Wolff, M.; Yang, R.; Ma, Y.; Aycardi, E. Fremanezumab for the Preventive Treatment of Chronic Migraine. N. Engl. J. Med. 2017, 377, 2113-2122. [CrossRef] [PubMed]

52. Brandes, J.L.; Kudrow, D.; Yeung, P.P.; Sakai, F.; Aycardi, E.; Blankenbiller, T.; Grozinski-Wolff, M.; Yang, R.; Ma, Y. Effects of fremanezumab on the use of acute headache medication and associated symptoms of migraine in patients with episodic migraine. Cephalalgia 2020, 40, 470-477. [CrossRef] [PubMed]

53. Ferrari, M.D.; Diener, H.C.; Ning, X.; Galic, M.; Cohen, J.M.; Yang, R.; Mueller, M.; Ahn, A.H.; Schwartz, Y.C.; Grozinski-Wolff, M.; et al. Fremanezumab versus placebo for migraine prevention in patients with documented failure to up to four migraine preventive medication classes (FOCUS): A randomised, double-blind, placebo-controlled, phase 3b trial. Lancet 2019, 394, 1030-1040. [CrossRef]

54. Goadsby, P.J.; Silberstein, S.D.; Yeung, P.P.; Cohen, J.M.; Ning, X.; Yang, R.; Dodick, D.W. Long-term safety, tolerability, and efficacy of fremanezumab in migraine: A randomized study. Neurology 2020, 95, e2487-e2499. [CrossRef] [PubMed] 
55. Stauffer, V.L.; Dodick, D.W.; Zhang, Q.; Carter, J.N.; Ailani, J.; Conley, R.R. Evaluation of Galcanezumab for the Prevention of Episodic Migraine: The EVOLVE-1 Randomized Clinical Trial. JAMA Neurol. 2018, 75, 1080-1088. [CrossRef]

56. Skljarevski, V.; Matharu, M.; Millen, B.A.; Ossipov, M.H.; Kim, B.-K.; Yang, J.Y. Efficacy and safety of galcanezumab for the prevention of episodic migraine: Results of the EVOLVE-2 Phase 3 randomized controlled clinical trial. Cephalalgia 2018, 38, 1442-1454. [CrossRef]

57. Detke, H.C.; Goadsby, P.J.; Wang, S.; Friedman, D.I.; Selzler, K.J.; Aurora, S.K. Galcanezumab in chronic migraine. Neurology 2018, 91, e2211. [CrossRef] [PubMed]

58. Camporeale, A.; Kudrow, D.; Sides, R.; Wang, S.; Van Dycke, A.; Selzler, K.J.; Stauffer, V.L. A phase 3, long-term, open-label safety study of Galcanezumab in patients with migraine. BMC Neurol. 2018, 18, 188. [CrossRef]

59. Stauffer, V.L.; Wang, S.; Voulgaropoulos, M.; Skljarevski, V.; Kovacik, A.; Aurora, S.K. Effect of Galcanezumab Following Treatment Cessation in Patients With Migraine: Results From 2 Randomized Phase 3 Trials. Headache J. Head Face Pain 2019, 59, 834-847. [CrossRef]

60. Mulleners, W.; Kim, B.-K.; Lainez, M.; Lanteri-Minet, M.; Aurora, S.; Nichols, R.; Wang, S.; Tockhorn-Heidenreich, A.; Detke, H. A Randomized, Placebo-Controlled Study of Galcanezumab in Patients with Treatment-Resistant Migraine: Double-Blind Results from the CONQUER Study (162). Neurology 2020, 94, 162.

61. Smith, T.R.; Janelidze, M.; Chakhava, G.; Cady, R.; Hirman, J.; Allan, B.; Pederson, S.; Smith, J.; Schaeffler, B. Eptinezumab for the Prevention of Episodic Migraine: Sustained Effect Through 1 Year of Treatment in the PROMISE-1 Study. Clin. Ther. 2020, 42, 2254-2265.e2253. [CrossRef] [PubMed]

62. Silberstein, S.; Diamond, M.; Hindiyeh, N.A.; Biondi, D.M.; Cady, R.; Hirman, J.; Allan, B.; Pederson, S.; Schaeffler, B.; Smith, J. Eptinezumab for the prevention of chronic migraine: Efficacy and safety through 24 weeks of treatment in the phase 3 PROMISE-2 (Prevention of migraine via intravenous ALD403 safety and efficacy-2) study. J. Headache Pain 2020, 21, 120. [CrossRef] [PubMed]

63. Kudrow, D.; Cady, R.K.; Allan, B.; Pederson, S.M.; Hirman, J.; Mehta, L.R.; Schaeffler, B.A. Long-term safety and tolerability of eptinezumab in patients with chronic migraine: A 2-year, open-label, phase 3 trial. BMC Neurol. 2021, 21, 126. [CrossRef] [PubMed]

64. Scuteri, D.; Corasaniti, M.T.; Tonin, P.; Bagetta, G. Eptinezumab for the treatment of migraine. Drugs Today 2019, 55, 695-703. [CrossRef] [PubMed]

65. Baker, B.; Schaeffler, B.; Beliveau, M.; Rubets, I.; Pederson, S.; Trinh, M.; Smith, J.; Latham, J. Population pharmacokinetic and exposure-response analysis of eptinezumab in the treatment of episodic and chronic migraine. Pharmacol. Res. Perspect. 2020, 8, e00567. [CrossRef]

66. Garcia-Martinez, L.F.; Raport, C.J.; Ojala, E.W.; Dutzar, B.; Anderson, K.; Stewart, E.; Kovacevich, B.; Baker, B.; Billgren, J.; Scalley-Kim, M.; et al. Pharmacologic Characterization of ALD403, a Potent Neutralizing Humanized Monoclonal Antibody Against the Calcitonin Gene-Related Peptide. J. Pharmacol. Exp. Ther. 2020, 374, 93. [CrossRef]

67. Scuteri, D.; Adornetto, A.; Rombolà, L.; Naturale, M.D.; Morrone, L.A.; Bagetta, G.; Tonin, P.; Corasaniti, M.T. New Trends in Migraine Pharmacology: Targeting Calcitonin Gene-Related Peptide (CGRP) With Monoclonal Antibodies. Front. Pharm. 2019, 10, 363. [CrossRef]

68. Mavridis, T.; Deligianni, C.I.; Karagiorgis, G.; Daponte, A.; Breza, M.; Mitsikostas, D.D. Monoclonal Antibodies Targeting CGRP: From Clinical Studies to Real-World Evidence-What Do We Know So Far? Pharmaceuticals 2021, 14, 700. [CrossRef]

69. Winner, P.K.; McAllister, P.; Chakhava, G.; Ailani, J.; Ettrup, A.; Krog Josiassen, M.; Lindsten, A.; Mehta, L.; Cady, R. Effects of Intravenous Eptinezumab vs Placebo on Headache Pain and Most Bothersome Symptom When Initiated During a Migraine Attack: A Randomized Clinical Trial. JAMA 2021, 325, 2348-2356. [CrossRef]

70. Drellia, K.; Kokoti, L.; Deligianni, C.I.; Papadopoulos, D.; Mitsikostas, D.D. Anti-CGRP monoclonal antibodies for migraine prevention: A systematic review and likelihood to help or harm analysis. Cephalalgia 2021, 41, 851-864. [CrossRef]

71. Scuteri, D.; Piro, B.; Morrone, L.A.; Corasaniti, M.T.; Vulnera, M.; Bagetta, G. The need for better access to pain treatment: Learning from drug consumption trends in the USA. Funct. Neurol. 2017, 22, 229-230. [CrossRef] [PubMed]

72. Scuteri, D.; Garreffa, M.R.; Esposito, S.; Bagetta, G.; Naturale, M.D.; Corasaniti, M.T. Evidence for accuracy of pain assessment and painkillers utilization in neuropsychiatric symptoms of dementia in Calabria region, Italy. Neural Regen. Res. 2018, 13, 1619-1621. [CrossRef]

73. Scuteri, D.; Vulnera, M.; Piro, B.; Bossio, R.B.; Morrone, L.A.; Sandrini, G.; Tamburin, S.; Tonin, P.; Bagetta, G.; Corasaniti, M.T. Pattern of treatment of behavioural and psychological symptoms of dementia and pain: Evidence on pharmacoutilization from a large real-world sample and from a centre for cognitive disturbances and dementia. Eur. J. Clin. Pharmacol. 2021, 77, 241-249. [CrossRef] [PubMed]

74. Mitsikostas, D.D.; Moskowitz, M.A. Making headway-A role for CGRP in post-traumatic headache. Nat. Rev. Neurol. 2021, 17, 133-134. [CrossRef] 